\title{
XEMIS2: A liquid xenon Compton camera to image small animals
}

\author{
Yuwei Zhu * \\ SUBATECH \\ IMT Atlantique, CNRS/IN2P3, \\ Université de Nantes \\ Nantes, France \\ * Corresponding author. \\ E-mail address: \\ Yuwei.Zhu@subatech.in2p3.fr \\ Julien Bert \\ INSERM \\ UMR1101, LATIM, CHRU \\ Morvan \\ Brest, France \\ Michel Cherel \\ INSERM U892 équipe 13 \\ Nantes, France

Jérôme Idier
LS2N
Ecole Centrale de Nantes,
CNRS/Inp, Université de Nantes
Nantes, France
Julien Masbou
SUBATECH
IMT Atlantique, CNRS/IN2P3,
Université de Nantes
Nantes, France
Dimitris Visvikis
INSERM
UMR1101, LaTIM, CHRU
Morvan
Brest, France

\author{
Stéphane Acounis \\ SUBATECH \\ IMT Atlantique, CNRS/IN2P3, \\ Université de Nantes \\ Nantes, France
}

\author{
Nicolas Beaupère \\ SUBATECH \\ IMT Atlantique, CNRS/IN2P3, \\ Université de Nantes \\ Nantes, France
}

\author{
Jean-Luc Beney \\ SUBATECH \\ IMT Atlantique, CNRS/IN2P3, \\ Université de Nantes \\ Nantes, France
}

\author{
Stéphane Bouvier \\ SUBATECH \\ IMT Atlantique, CNRS/IN2P3, \\ Université de Nantes \\ Nantes, France \\ Jean-Pierre Cussonneau \\ SUBATECH \\ IMT Atlantique, CNRS/IN2P3, \\ Université de Nantes \\ Nantes, France
}

Françoise Kraeber-Bodéré

Centre Hospitalier

Universitaire de Nantes

Nantes, France

\author{
Eric Morteau \\ SUBATECH \\ IMT Atlantique, CNRS/IN2P3, \\ Université de Nantes \\ Nantes, France \\ Yajing Xing \\ SUBATECH \\ IMT Atlantique, CNRS/IN2P3, \\ Université de Nantes \\ Nantes, France
}

\author{
Clotilde Canot \\ SUBATECH \\ IMT Atlantique, CNRS/IN2P3, \\ Université de Nantes \\ Nantes, France
}

Sara Diglio

SUBATECH

IMT Atlantique, CNRS/IN2P3,

Université de Nantes

Nantes, France

Patrick Le Ray

SUBATECH

IMT Atlantique, CNRS/IN2P3,

Université de Nantes

Nantes, France

Jean-Sébastien Stutzmann

SUBATECH

IMT Atlantique, CNRS/IN2P3,

Université de Nantes

Nantes, France
Thomas Carlier

Centre Hospitalier

Universitaire de Nantes

Nantes, France

Debora Giovagnoli

INSERM

UMR1101, LATIM, CHRU

Morvan

Brest, France

Frédéric Lefèvre

SUBATECH

IMT Atlantique, CNRS/IN2P3,

Université de Nantes

Nantes, France

Dominique Thers

SUBATECH

IMT Atlantique, CNRS/IN2P3,

Université de Nantes

Nantes, France

\footnotetext{
Abstract - An innovative Xenon Medical Imaging System, XEMIS2, designed for small animal $3 \gamma$ preclinical imaging, has been constructed, and it is currently under test and qualification at the SUBATECH laboratory. It consists of a Compton camera, containing nearly $200 \mathrm{~kg}$ of liquid xenon, whose main goals are the precise three-dimensional localization of the ${ }^{44} \mathrm{Sc}$ radioactive emitter used to image the small animal and the reduction of the administered radiopharmaceutical activity in cancer diagnosis. The active volume of the XEMIS2 camera is surrounded by a set of PhotoMultiplier Tubes (PMTs) to measure scintillation light. The read-out anodes are segmented in $\mathbf{2 0 0 0 0}$ pixels to measure ionization charges. In order to reduce the electronics dead-time during continuous data taking, a novel DAQ system specifically designed for XEMIS2 has been realized and recently tested. It consists of two independent synchronized scintillation and ionization signal detection chains. The self-triggered scintillation light detection chain has been recently tested and calibrated in XEMIS1, whose experimental results showed a good performance. XEMIS2 will be soon installed at the Center for Applied Multimodal Imaging (CIMA) in the Nantes University Hospital for further preclinical studies. To safely
}

manage a large amount of xenon in a hospital center, a recovery and storage cryogenic subsystem called ReStoX has been conceived, successfully commissioned, and already installed at CIMA.

Keywords - Liquid xenon, Compton telescope, 3 gamma imaging, Scintillation light, Functional imaging.

\section{INTRODUCTION}

In recent decades, the functional imaging technique used in nuclear medicine has demonstrated significant value in tumor diagnosis. The reduction of the administered radiopharmaceutical activity to the patient, the personalized medicine by mean of therapeutic follow-up and the shortening of the exposure time are three important indicators to guide the future improvements for nuclear medicine imaging.

In order to obtain a good quality image with a significant reduction of the administered activity to the patient for oncology diagnosis, we propose an innovative low dose functional imaging technique called $3 \gamma$ imaging. This 
technique consists in combining a large Field Of View (FOV) liquid xenon (LXe) Compton camera with a 3D localization of the radioactive decay of a specific $\left(\beta^{+}, \gamma\right)$ radionuclide emitter, the scandium-44 $\left({ }^{44} \mathrm{Sc}\right)[1]$. Furthermore, innovative radiopharmaceuticals are under study at SUBATECH laboratory, ARRONAX GIP, CRCINA, and Unité AMaROC ONIRIS at Nantes [2].

The positron and the third $\gamma$-ray are emitted by the ${ }^{44} \mathrm{Sc}$ in time and space quasi-coincidences. The intersection between the reconstructed Line Of Response (LOR) and the Compton cone allows the localization of the radionuclide, where the LOR is determined by the coincidence detection of the two back-to-back $511 \mathrm{keV} \gamma$-rays resulting from the positron annihilation with an electron, and the Compton cone is given by the interaction of the third $\gamma$-ray in the Compton camera $[3,4]$. Reducing the administered activity and providing a high-resolved $3 \mathrm{D}$ image at the same time, can be possible if a Compton camera with a high scattering efficiency and high energy and spatial resolutions is used [5]. A Liquid Xenon Time Projection Chamber (LXeTPC) has proven to be a perfect candidate for these purposes in a wide energy range from several tens of $\mathrm{keV}$ to tens of $\mathrm{MeV}$ [6].

The first prototype of the XEMIS project, XEMIS1, has successfully proved the feasibility of single-phase LXeTPC as Compton camera. XEMIS1 consists of a TPC of $2.8 \mathrm{~cm} \times 2.8$ $\mathrm{cm} \times 12 / 6 \mathrm{~cm}$ active volume filled with LXe. To characterize the detector performance, XEMIS1 has been calibrated with the $511 \mathrm{keV} \gamma$-ray from a low activity sodium-22 $\left({ }^{22} \mathrm{Na}\right)$ source, which is also a $\left(\beta^{+}, \gamma\right)$ emitter. This first prototype has shown a $5 \%$ energy resolution and a timing resolution of $44.3 \pm 3.0 \mathrm{~ns}$ for $511 \mathrm{keV}$ photoelectric events under an electric field of $1 \mathrm{kV} / \mathrm{cm}$, which is equivalent to a spatial resolution along the $\mathrm{z}$-axis (i.e., in electric field direction) of roughly 100 $\mu \mathrm{m}[4]$.

In the following, we will focus on XEMIS2. This paper is organized as follows: Section 2 details the characteristics of XEMIS2; the new cryogenic subsystem ReStoX is presented in Section 3; the readout electronics for the measurement of the scintillation signal and some preliminary results are presented in Section 4; finally, Section 5 is devoted to conclusions.

\section{XEMIS2}

To demonstrate the advantages of the $3 \gamma$ imaging, a new large Compton camera XEMIS2 for small animal imaging has been designed. The XEMIS2 facility consists of three main sub-systems: the XEMIS2 cryostat, the purification and recirculation systems, and the recovering and storage cryogenic subsystem (ReStoX).

The XEMIS2 cryostat is designed as a monolithic and highly sensitive cylindrical camera containing nearly $200 \mathrm{~kg}$ of LXe, with a geometrical acceptance for small animals ranging between $50 \%$ and $75 \%$ from the edge to the center of the FOV. This camera contains two identical back-to-back LXeTPCs, $12 \mathrm{~cm}$ drift length each. A cutout view of the design of XEMIS2 is presented in Fig.1. When a $\gamma$-ray interacts with LXe, since the scintillation light emission in LXe is a fast process (of the order of several tens of ns), the detection of the Vacuum Ultra Violet (VUV) scintillation photons by the PhotoMultiplier Tube (PMT) provides the initial time of the event $t_{0}$. The ionized electrons are drifted towards the segmented anodes under a homogeneous electric field and finally form the ionization signal, that permits to get the $\mathrm{X}-\mathrm{Y}$ position and deposited energy of each interaction vertex. A high drift field is needed to reduce the electrons/ions recombination process for a precise ionization signal measurement. On the other hand, the high drift field reduces the VUV scintillation photon production. XEMIS2 operates under a drift field of $2 \mathrm{kV} / \mathrm{cm}$, whose corresponding scintillation yield is around 20000 photons/MeV [7].

To detect the VUV scintillation photons, in a preliminary stage, 64 PMTs are distributed around the active volume of XEMIS2. In the near future, the surface of the active area will be completely covered by 380 PMTs [8]. Two segmented anodes are located on both sides, and a shared cathode is placed in the center of the XEMIS2 camera. These two anodes are segmented into 20000 pixels with ultra-low noise electronic read-out [9] to detect the ionized charges produced after the interaction of ionizing radiation. A preliminary full GATE/Geant4 [10] simulation showed that it is possible to obtain a good quality image of a small animal with only 20 $\mathrm{kBq}$ of ${ }^{44} \mathrm{Sc}$ in 20 minutes of exposure $[11,12]$.

\section{RESTOX}

Since the XEMIS2 camera has been conceived to be installed in a hospital center, its design needs to be compact and meet the safety requirements for operations. For this reason, a high-pressure closed-loop cryogenic subsystem named ReStoX (Recovery and Storage system of Xenon) has been designed and built by the AIR LIQUIDE Advanced Technologies Division and SUBATECH laboratory. The XEMIS2 LXe cryogenic installation can be seen in Fig. 2.

ReStoX is a double-walled insulated stainless-steel tank, which can store up to about $200 \mathrm{~kg}$ of xenon from room

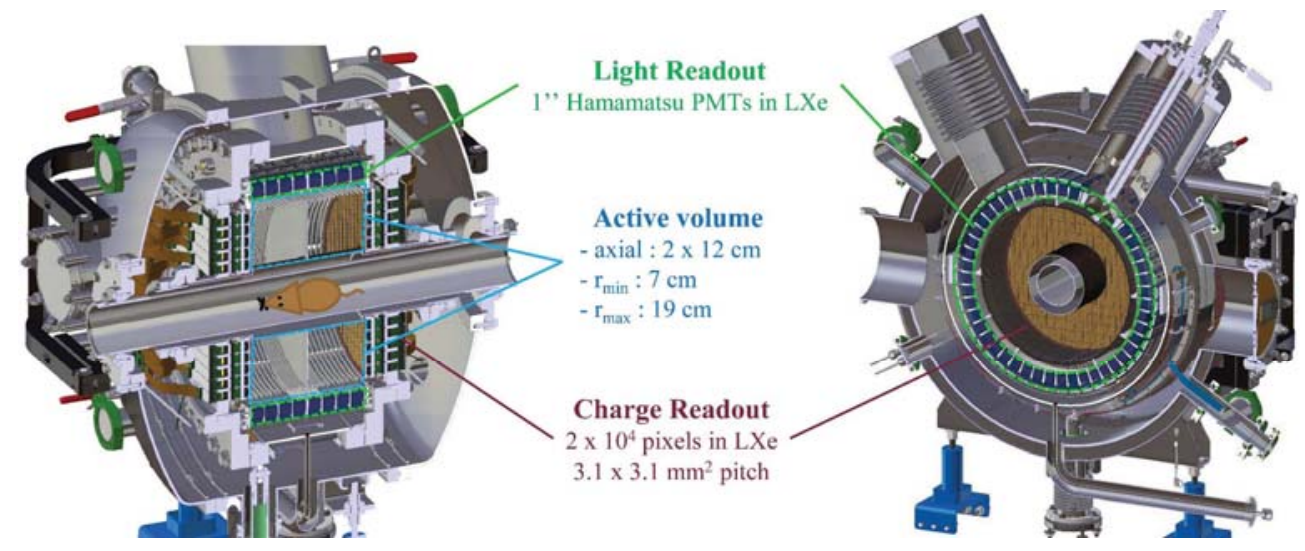

Fig. 1. Design of the XEMIS2 camera 


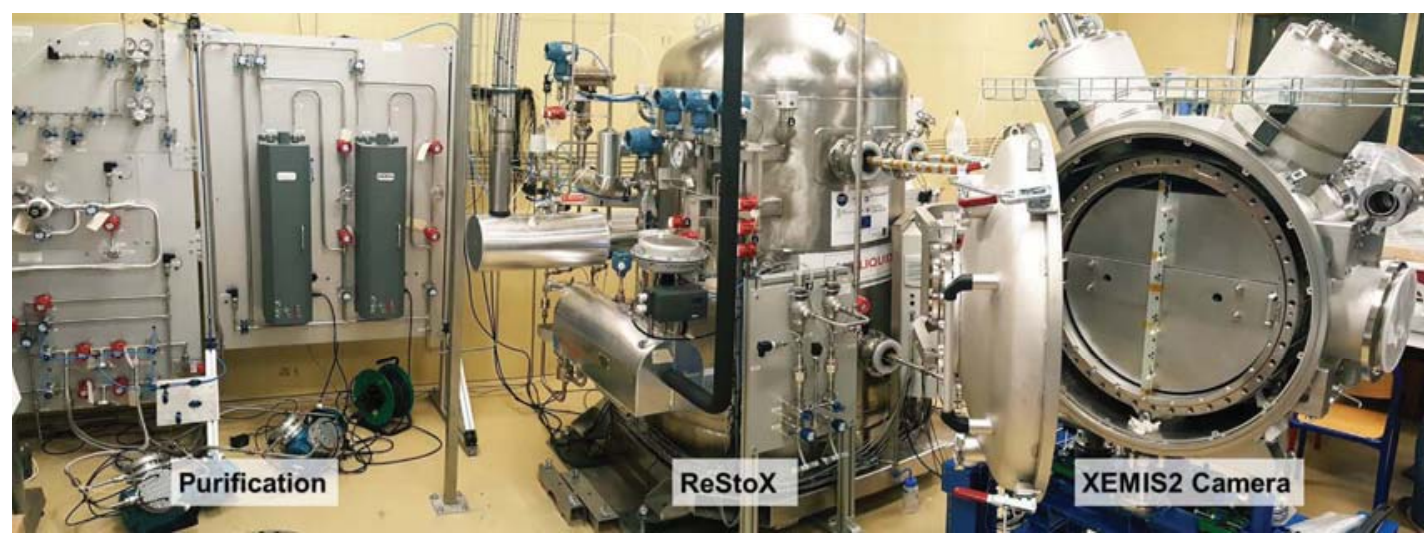

Fig. 2. Overview of the XEMIS2 cryogenics facility

temperature to liquid xenon temperature $(168 \mathrm{~K})$. Furthermore, the liquid nitrogen circulation in a specifically designed aluminum block located inside the ReStoX tank provides a high cooling power of $10 \mathrm{~kW}$, that contributes to a high cold xenon handling efficiency [11]. Experimental results showed that in case of cooling source loss, the temperature would rise from LXe temperature to room temperature in almost 1 year [13].

The commissioning of the whole cryogenic infrastructure has been carried out for $128 \mathrm{~kg}$ of LXe in several operation stages. The stability of the LXe temperature and pressure inside the camera has been measured. Moreover, it has been proven that in case of emergency, a fast and complete passive recovery of the full xenon in liquid state from the XEMIS2 cryostat to the ReStoX tank could be realized in less than 10 minutes through a gravity assisted method [13].

\section{SCINTILLATION Light MEASUREMENTS}

The importance of scintillation signals in XEMIS2 comes from several aspects: in the first place, these signals can provide the $\gamma$-rays interaction time. While the X-Y position of each interaction is determined by the ionization signals, it is possible to combine scintillation and ionization signals to reconstruct the $\mathrm{Z}$ position of each interaction with a $100 \mu \mathrm{m}$ resolution. To reach such accuracy, the time resolution of scintillation light needs to be below 50 ns. Furthermore, the scintillation signals can be used for spatial pre-localization of $\gamma$-ray interactions to optimize the position reconstruction algorithm by combining both scintillation and ionization signals. Additionally, in the case of need to increase the administered activity to shorten the time of imaging, the prelocalization with scintillation signal can help to reduce the occupancy of the TPC due to the nonnegligible drift time of the charge carriers up to $60 \mu \mathrm{s}$.

In order to have a continuous read-out, with negligible dead-time during 20 minutes, a novel higher capability Data acquisition (DAQ) system dedicated to XEMIS2 is developed, and it is currently under qualification. This DAQ consists of two independent synchronized scintillation and ionization signal detection chains. The scintillation light detection chain is composed of four parts: PMT, pulse-shaping amplifier, discriminator, and XEMIS Data Concentrator (XDC).

\section{A. Novel Scintillation Light Detection Chain}

The VUV-sensitive Hamamatsu R7600-06MOD-ASSY PMTs have been chosen to detect the scintillation light at 178 $\mathrm{nm}$ wavelength. This type of PMT with a minimum active area of $18 \times 18 \mathrm{~mm}^{2}$ is specially designed to work at 1.2 bar and
$168 \mathrm{~K}$ in the LXe. It shows a good VUV scintillation photon sensitivity with a quantum efficiency of $35 \%$ at $178 \mathrm{~nm}$. To obtain a homogeneous response of the physical signal for different PMTs, the gain of each PMT needs to be calibrated, meaning that each PMT needs to be provided with the suitable high-voltage power supply.

To measure the interaction time and the number of VUV scintillation photons produced in LXe for each gamma interaction, the output pulses of each PMT need to be filtered, shaped, and amplified. For this purpose, a pulse-shaping amplifier has been developed. It consists of a sixth-order RLC low-pass filter that was inspired by the work of the ATLAS Tile Calorimeter [14]. The output shaped pulses were simulated by considering the effects of different electronic components. Since the scintillation light of LXe is emitted with an average decay time of around $30 \mathrm{~ns}$ for an electric field of $2 \mathrm{kV} / \mathrm{cm}(3 \%$ singlet excited state with decay time of 2.2 ns, $60 \%$ triplet excited state with $27 \mathrm{~ns}$ and $37 \%$ recombination about $45 \mathrm{~ns}$ for an electric field of $2 \mathrm{kV} / \mathrm{cm}$ [15]), the scintillation photons are not produced simultaneously. Under real circumstances, the duration time of the output pulse generated by PMTs in XEMIS1 is of the order of $50 \mathrm{~ns}$. Therefore, the pulse-shaping amplifier integrates effectively the signal generated by the PMT with a peaking time of about $50 \mathrm{~ns}$, as presented in Fig. 3. A better integration could be achieved if the peaking time was longer, nevertheless we estimate the current achievement to be a good compromise to reduce the detector occupancy effectively.

No additional global trigger system for physic events selection is present in XEMIS2. For the scintillation light measurement, to select the physical events, a threshold on the pulse height after the pulse-shaping amplifier is needed for each self-triggered PMT channel. In this way, shaped linear pulses are converted into logic pulses by the discriminator. After studying several methods of VUV scintillation photon time measurements and considering the R\&D constraints, the combination of pulse-shaping amplifier and discriminator module was chosen to measure the initial time of the $\gamma$-ray event. Since a low cost electronic is required, we decided to measure the pulse duration between the leading edge and trailing edge of the discriminator (i.e., the Time Over Threshold (TOT)) to estimate the number of photoelectrons detected by the PMT. Finally, the data flow of 32 PMT channels of each half TPC is concentrated on the FieldProgrammable Gate Array (FPGA) of the XDC. A large amount of data produced by the low threshold self-triggered electronics is treated afterward. 


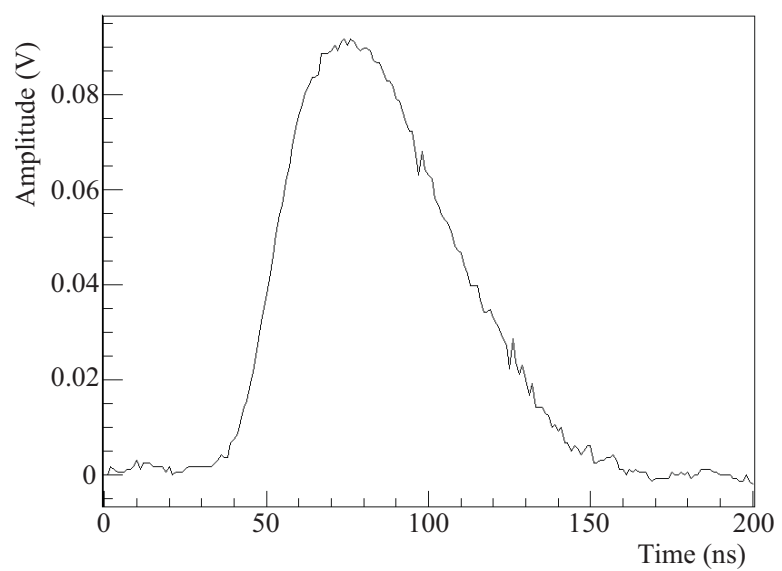

Fig. 3. Example of output pulse generated by pulse-shaping amplifier with injected charges

\section{B. Calibration of Scintillation Light Detection Chain}

A detailed study of the electronics response of each part of the scintillation signal detection chain is necessary to characterize the detector performances. During the first test in XEMIS1, the scintillation light detection chain has been successfully tested and qualified. To calibrate the PMTs, a Light Emitting Diode (LED) connected with an optical fiber which extends to the inside of the detector is used to illuminate the PMTs. This setup allows the determination and monitoring of the PMT gain in situ. The results showed that with the increase of high-voltage power supply of the PMT, the gain varies exponentially. In the calibration tests of the pulseshaping amplifier and the discriminator, the injected charges correspond to a given number of photoelectrons with the PMT gain of $10^{6}$. The pulse-shaping amplifier of each channel has good electronic linearity. In addition, there is a dependence of the TOT on the number of photoelectrons, as presented in Fig.4. In the case of few photoelectrons collection (likely situation in XEMIS2), the TOT varies as expected, meaning that the number of collected photoelectrons is easily distinguishable from the TOT. A systematic study at LXe temperature is in progress.

\section{CONCLUSION}

XEMIS2 is under test and qualification to prove that a LXe Compton camera combined with the $3 \gamma$ imaging technique is a potentially good candidate for cancer diagnosis. A significant reduction of the activity administrated to the

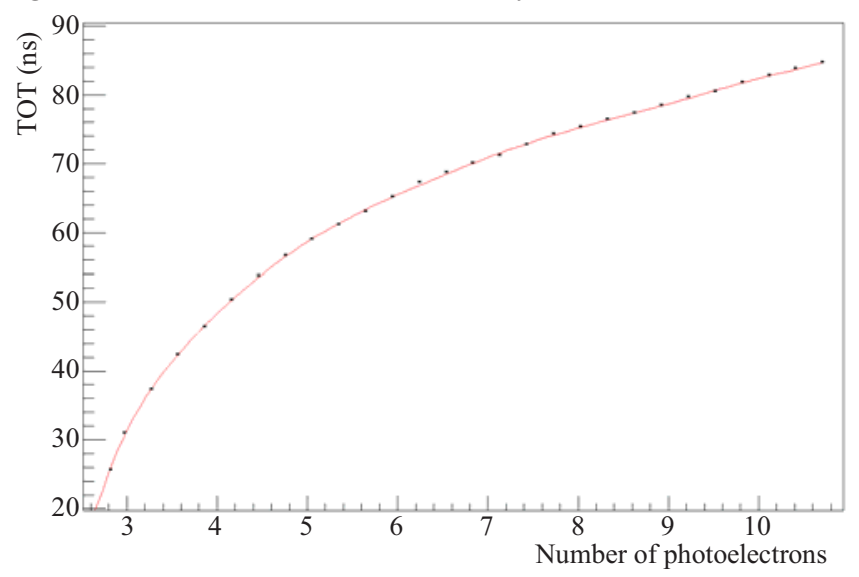

Fig. 4. Dependence of the Time Over Threshold (TOT) on the number of photoelectrons with a threshold of 2 photoelectrons patient is foreseen while preserving the image quality of standard PET imaging. Lots of innovations were brought into the design and construction of XEMIS2 for both camera and cryogenic subsystem. The assembly of XEMIS2 is ongoing at SUBATECH laboratory while all electronic chains for ionization and scintillation signals detection are under qualification. The preliminary results of calibration tests showed a good performance for the scintillation light detection chain. For further preclinical studies, XEMIS2 will be soon installed in a Center for Applied Multimodal Imaging at Nantes University Hospital.

\section{ACKNOWLEDGMENTS}

The research presented in this paper has been funded by the E.U., the Region Pays de la Loire in France and by grants from the French National Agency for Research, "Investisement d'Avenir' ArronaxPlus Equipex no ANR-11EQPX-0004.

\section{REFERENCES}

[1] S. Huclier-Markai, et al., Optimization of reaction conditions for the radiolabeling of DOTA and DOTA-peptide with ${ }^{44 \mathrm{~m} / 44} \mathrm{sc}$ and experimental evidence of the feasibility of an in vivo PET generator, Nucl. Med. Biol. 41 (2014) 36-43.

[2] S. Huclier-Markai, et al., Promising Scandium Radionuclides for Nuclear Medicine: A Review on the Production and Chemistry up to In Vivo Proofs of Concept, Cancer biotherapy \& radiopharmaceuticals 33.8 (2018): 316-329.

[3] R. W. Todd, J. M. Nightingale, and D. B. Everett, A proposed $\gamma$ camera, Nature 251.5471 (1974) 132-134.

[4] Y. Xing, et al., XEMIS: Liquid Xenon Compton Camera for $3 \gamma$ Imaging, 4th International Conference on Technology and Instrumentation in Particle Physics, Springer Proc. Phys. 213 (2017) 154-158.

[5] C. Lang, D. Habs, K. Parodi, and P.G. Thirolf, Sub-millimeter nuclear medical imaging with high sensitivity in positron emission tomography using $\beta+\gamma$ coincidences, Journal of Instrumentation 9.01 (2014): P01008.

[6] E. Aprile, and T. Doke, Liquid Xenon Detectors for Particle Physics and Astrophysics, Reviews of Modern Physics 82.3 (2010) 2053-2097.

[7] T. Doke, et al., Absolute Scintillation Yields in Liquid Argon and Xenon for Various Particles, Jap. J. Appl. Phys. 41.3R (2002) 15381545 .

[8] Y. Zhu, et al., Scintillation Signal in XEMIS2, a Liquid Xenon Compton Camera with $3 \gamma$ Imaging Technique, 4th International Conference on Technology and Instrumentation in Particle Physics, Springer Proc. Phys. 213 (2017) 159-163.

[9] O. Lemaire, et al., Development of a Readout Electronic for the Measurement of Ionization in Liquid Xenon Compton Telescope Containing Micro-patterns, IEEE Nuclear Science Symposium Conference (NSS/MIC) (2012): 858-861.

[10] S. Jan, et al., GATE: a simulation toolkit for PET and SPECT, Phys. Med. Biol. 49 (2004) 4543-4561.

[11] J.P. Cussonneau, et al., $3 \gamma$ Medical Imaging with a Liquid Xenon Compton Camera and ${ }^{44}$ Sc Radionuclide, Acta Phys. Pol. B Vol. 48, No. 10, Oct. (2017).

[12] L. Gallego Manzano, et al., XEMIS2: A liquid xenon detector for small animal medical imaging, Nucl. Instrum. Methods A 912 (2018) 329_ 332.

[13] L. Virone, et al., Gravity assisted recovery of liquid xenon at large mass flow rates, Nucl. Instrum. Methods A 893(2018) 10-14.

[14] F. Tang, et al., Design of the Front-end Readout Electronics for ATLAS Tile Calorimeter at the sLHC, 17th IEEE-NPSS Real Time Conference (2010) 1-5.

[15] S. Kubota, M. Hishida, M. Suzuki, and J.Z.R. Gen, Dynamical behavior of free electrons in the recombination process in liquid argon, krypton, and xenon, Physical Review B 20.8 (1979) 3486-3496. 